"Hasta que valga la pena vivir": violencia y reproducción social como claves de la emergencia feminista contemporánea en América Latina

Luna Follegati - Pierina Ferretti

Anuario No 32 / ISSN 1853-8835 / 2020

http://anuariodehistoria.unr.edu.ar/ojs/index.php/Anuario/index

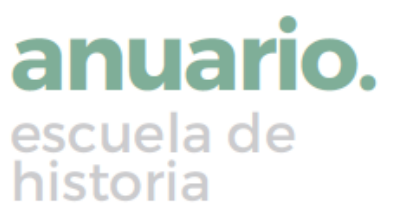

\title{
"Hasta que valga la pena vivir": violencia y reproducción social como claves de la emergencia feminista contemporánea en América Latina
}

\author{
"Until living becomes worth it": violence and social \\ reproduction as keys to Latin American contemporary \\ feminist rise
}

LUNA FOLLEGATI MONTENEGRO

Universidad de Chile (Chile)

lfollegatti@gmail.com

PIERINA FERRETTI

Centro de Estudios Culturales Latinoamericanos, Facultad de Filosofia y Humanidades, Universidad de Chile (Chile) pierinaferretti@gmail.com

RESUMEN

El artículo ofrece una lectura sobre la emergencia feminista contemporánea en América Latina. La hipótesis que se plantea es que los feminismos actuales en el continente se aglutinan en torno al tema de la violencia contra las mujeres y cuerpos feminizados. Esta centralidad de la violencia se debería, por una parte, a la importancia de la trayectoria histórica que ha tenido esta temática en la historia reciente vinculada a las dictaduras y procesos autoritarios, y, por otra, a las condiciones de recrudecimiento de la violencia contra las mujeres y cuerpos feminizados en un escenario de neoliberalismo avanzado y crisis de la reproducción social. Se recorre el tratamiento que el movimiento feminista ha dado a la problemática de la violencia contra las mujeres y cuerpos feminizados tanto en su sentido específico como en sus dimensiones estructurales, destacando el rol articulador a nivel histórico y contemporáneo. Además, se aborda la reflexión sobre la violencia en clave estructural y el problema de la reproducción social en un contexto de crisis de los cuidados y de luchas y conflictos asociados a estas dimensiones de la vida social. En la conclusión se plantean perspectivas críticas y aperturas que presenta el movimiento en la actualidad. 

claves de la emergencia feminista contemporánea en América Latina

Palabras clave: Movimiento feminista; violencia de género; crisis de cuidado; violencia estructural; teoria feminista.

\section{ABSTRACT}

The article offers a reading on the contemporary feminist rise in Latin America. The hypothesis is that current feminisms in the continent are gathered around the issue of violence against women and feminized bodies. This centrality of violence is due, on the one hand, to the importance of the historical trajectory that this theme has had in recent history, linked to dictatorships and authoritarian processes, and, on the other hand, to the conditions of intensification of violence against women and feminized bodies in a scenario of advanced neoliberalism and crisis of social reproduction. It reviews the treatment that the feminist movement has given to the problem of violence against women and feminized bodies both in its specific sense and in its structural dimensions, highlighting the articulating role at the historical and contemporary level. It also addresses the reflection on violence in a structural key and the problem of social reproduction in a context of crisis of care and struggles and conflicts associated with these dimensions of social life. In the conclusion, critical perspectives and openings presented by the movement today are raised.

Keywords: Feminist movement; gender violence; care crisis; structural violence; feminist theory.

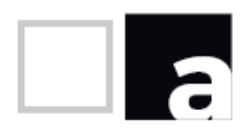

\section{Introducción}

"Cuarta ola", "marea verde" o "revolución feminista" son algunos de los modos en que se ha nombrado la emergencia contemporánea de movimientos de mujeres sorprendentemente masivos en distintos puntos del globo en los últimos cinco años. Más allá de las denominaciones provisorias, resulta insoslayable que estamos ante un fenómeno nuevo, que si bien hunde sus raíces en la historia larga del movimiento feminista y sus anteriores momentos de irrupción masiva en el espacio público, constituye en sí mismo un episodio particular con caracteristicas propias, que, por una parte, dan continuidad a demandas feministas de larga data y, por otra, amplian el radio de acción y de intervención feminista a esferas de la vida social cada vez mayores,

\section{anuario.}


$\overline{\text { incorporando cuestionamientos a la política financiera, la ecología, el }}$ extractivismo y los sistemas de seguridad social, por poner algunos ejemplos.

Con los limites que tiene todo intento de fechar procesos que se fraguan durante décadas, podríamos situar el comienzo de este nuevo ciclo en el paro de mujeres convocado en Argentina en junio de 2015 como protesta ante el femicidio de la adolescente Chiara Páez, ocasión en la que, además, se popularizó la consigna "Ni Una Menos" que al dia de hoy es un grito global contra la violencia hacia las mujeres. La contundencia de ese acto, convocado y efectuado en pocos días y con una inusitada adhesión, indicaba una modificación social profunda hacia la violencia de género: el tránsito de la naturalización al rechazo, y de la victimización en el espacio privado a la agencia política en la esfera pública. La lucha contra la violencia se volvió, así, un motor capaz de movilizar enormes masas de mujeres, traspasando incluso fronteras geográficas. De igual manera, el movimiento feminista estudiantil chileno, cuyo despliegue se produjo entre los meses de abril y julio del 2018, destacó un importante aspecto al relevar reivindicaciones como la Educación no sexista y denunciando situaciones de acoso y abuso sexual en los espacios educativos, manifestaciones que se replicaron en otros países como México y Colombia (Mingo, 2020; Forero, 2019). En todos ellos, el contenido volvía a ser uno solo: no más violencia hacia las mujeres en la Universidad. A partir de allí, presenciamos una multiplicación de movilizaciones cada vez más masivas y transnacionales, como lo confirmó tanto el fenómeno global de la performance "Un violador en tu camino" que, iniciado en Chile por el colectivo Las Tesis, dio la vuelta al mundo (Follegati, 2020), como la inédita masividad que alcanzó la conmemoración del Día Internacional de las Mujeres Trabajadoras en marzo de 2020.

Siendo uno de los procesos sociales más relevantes de nuestra época, la caracterización precisa de esta emergencia feminista es compleja, porque en ella convive una heterogeneidad importante de perspectivas y una diversidad generacional, de clases y étnica que cuestiona las lecturas clásicas sobre los movimientos sociales (Tarrow, 1989; Melucci, 1996; Touraine, 1985). En las actuales movilizaciones de mujeres habita una diversidad enorme y en las calles pueden encontrarse desde feministas socialistas a feministas liberales, militantes políticas a mujeres sin militancia alguna y, al menos en Chile, la escala masiva de las manifestaciones feministas se ha debido precisamente a la movilización de mujeres que no necesariamente pertenecen a un colectivo, organización o partido. Conceptos como clase obrera (en su acepción tradicional asociada al proletariado industrial), conciencia de clase, contradicción primaria, o la polaridad izquierda-derecha, que fueron piezas clave de la caja de herramientas teóricas con las que se comprendió la conflictividad social y el antagonismo en el siglo XX, no alcanzan para dar cuenta de lo que está 
"Hasta que valga la pena vivir": violencia y reproducción social como claves de la emergencia feminista contemporánea en América Latina

ocurriendo con el movimiento feminista. Entretanto, los ensayos interpretativos que mejor capturan la novedad del momento presente recién están viendo la luz (Gago, 2019; Pleyers, 2018; Almeida, 2020). Sin embargo, hay un consenso en que la masividad de las movilizaciones que se han producido, su carácter transnacional y la centralidad que adquieren tanto el repudio a la violencia contra las mujeres como el derecho al aborto, son marcas de identidad que caracterizan esta emergencia, así como la constatación de la preponderante presencia de mujeres en las más importantes luchas contra el extractivismo y la desposesión de los bienes comunes, y en otras disputas que se libran en el terreno de la reproducción social. A modo de clave de lectura, es posible señalar que en la actualidad la articulación masiva y explosiva del feminismo ha estado vinculada a la necesidad de una respuesta inmediata frente a situaciones sociales urgentes, donde resaltamos dos ejes: la violencia extrema contra las mujeres y las condiciones de precarización de la vida producidas por el avance neoliberal en la región.

A partir de lo anterior, el objetivo del presente artículo es proponer algunas propuestas de lectura para comprender la emergencia actual del movimiento feminista en América Latina. En este sentido, es posible señalar que los feminismos de la historia reciente latinoamericana han situado el problema de la violencia contra la mujer de forma insistente en el plano político, cuestión que ha sido desarrollada tanto por la acción del movimiento feminista, como también por el posicionamiento de la temática en la agenda internacional. En la actualidad, uno de los aspectos gravitantes del movimiento ha estado vinculado a la activación y visibilización del problema a partir de consignas que explicitan situaciones como los femicidios, o la comprensión de la violencia como un continuo, a través de consignas como "ni una menos" y "vivas nos queremos". El carácter transversal de estas demandas en América Latina, señala la potencia contingente y actual que ha adquirido el feminismo en la región, relevando la importancia histórica de la lucha de las mujeres en esta materia. En este sentido, se vuelve ineludible referirnos a dos aspectos, tanto el carácter histórico de las demandas contra la violencia hacia las mujeres como también el potencial articulador que contiene el problema. Así, podría pensarse que la temática de la violencia ha sido un factor sustantivito para la articulación del movimiento feminista, pero también para la activación de su potencia y masividad actual. A modo de hipótesis para el presente artículo, es posible señalar que el factor aglutinante del eje violencia en los feminismos latinoamericanos actuales radicaría en dos aspectos. Por una parte, en la importancia de la trayectoria histórica que ha tenido esta temática en la historia reciente vinculada a las dictaduras y procesos autoritarios, aspecto asociado a la proliferación de organizaciones y colectivos sobre la materia. Por otra, en la problematización específica de la violencia contra las mujeres en clave estructural, es decir, como 
un problema que posibilita la comprensión de otros tipos de violencia, ya sea económica, política, institucional, laboral, etc. Así, el feminismo ha provisto a las mujeres de herramientas teóricas y prácticas para hacer frente a problemas urgentes del presente: la falta de derechos sexuales y reproductivos, la violencia de género y la crisis la reproducción social de la vida son los elementos como aspectos aglutinantes que explican el y factores sustantivos del carácter masivo que este ha adquirido durante los últimos años.

Conceptualmente, consideramos el término violencia a partir de las características desarrolladas por Segato (2003) y Osborne (2009), enfatizando que la violencia corresponde a un fenómeno estructural, considerando el amplio espectro que abarca desde la violencia sexual a situaciones acoso y malos tratos, fundamentadas en las relaciones de género cimentadas por el sistema patriarcal. En este sentido, la violencia contra las mujeres correspondería a un mecanismo de control y disciplinamiento, en tanto establece un lenguaje desde la violencia que responde a un principio que potencialmente expone a todas las mujeres, comprendiendo la violencia como un continuo, es decir, que "sería un extremo de conductas que se consideran normales" (Osborne, 2009, p. 16). La violencia contribuye a una estructura de diferenciación entre sujetos masculinos y femeninos, estableciendo una oposición y generando una estructura de relaciones de carácter simbólica donde lo masculino y femenino se articulan como posiciones relativas, más allá de las anatomías del hombre y la mujer (Segato, 2003). En este sentido, el sistema patriarcal configura en el cuerpo de las mujeres un espacio de control, dominación y subordinación, a través de la normalización de las jerarquías diferenciadoras de lo masculino y lo femenino. Por este motivo, nos referiremos a la violencia contra las mujeres y cuerpos feminizados explicitando así la necesidad de enfatizar en el acento de la violencia de género perpetrada desde el canon patriarcal, machista o sexista, y evidenciando además que las víctimas corresponden mayoritariamente a mujeres y cuerpos feminizados (Follegati, 2019). Así, concordamos con Rita Segato al señalar que los procesos de violencia articulan "estrategias de reproducción del sistema, mediante su refundación permanente, la renovación de los votos de subordinación de los minorizados en el orden de estatus, y el permanente ocultamiento del acto instaurador. Sólo así es posible advertir que estamos en una historia, la profundísima historia de la erección del orden de género y de su conservación por medio de una mecánica que rehace y revive su mito fundador todos los días" (Segato, 2003, p. 113).

El artículo está compuesto por cuatro apartados. El primero, "La emergencia histórica de los feminismos actuales", busca relevar la especificidad histórica de la emergencia feminista contemporánea ocurrida en un contexto global y regional determinados por el estado avanzado de la expansión neoliberal. Luego, 
"Hasta que valga la pena vivir": violencia y reproducción social como claves de la emergencia feminista contemporánea en América Latina

en "El nudo de la violencia como clave de articulación y reivindicación histórica", se recorre el tratamiento que desde el movimiento feminista se ha dado a la problemática de la violencia contra las mujeres, destacando el rol articulador que esta cuestión ha tenido en el desarrollo histórico del movimiento y en la adhesión masiva que concita en la actualidad. En "Violencia estructural y crisis de la reproducción social" se enfatiza en la mirada que el feminismo ha desarrollado en torno a las violencias estructurales y a partir de ella centrarnos en la reactivación de la reflexión sobre la reproducción social en el presente, en un contexto de crisis de los cuidados y de luchas y conflictos sociales asociados a estas cuestiones. En la conclusión, se plantean ejes críticos y aperturas sobre el desarrollo actual del movimiento feminista latinoamericano, así como potenciales proyecciones y desafios venideros.

\section{La emergencia histórica de los feminismos actuales}

El movimiento feminista posee un importante eje histórico y situado. Sus reivindicaciones, formas de organización, tendencias y despliegue se estructuran en relación a fuerzas globales en pugna, como también a las coyunturas políticas y sociales que atraviesan los Estados y contextos locales. En la actualidad, y a diferencia de las movilizaciones de los sesenta y setenta producidas en un escenario de ascenso de las luchas populares, la emergencia feminista ocurre en un momento de hegemonía neoliberal a escala planetaria y de un avance intensivo del capital sobre la vida, distanciándose del movimiento de la segunda ola (Schild, 2016). Los feminismos de los sesenta y setenta ocurrieron en un momento de crisis del orden social de la posguerra y de radicalización política, donde las luchas de liberación nacional, el black power, la oposición a la guerra de Vietnam, el mayo del 68' y una miriada de movimientos contestatarios en el campo cultural, se trenzaban con las luchas por el aborto y los derechos reproductivos, el salario para el trabajo doméstico, los movimientos de liberación homosexual y con los feminismos negros que introducian los elementos de raza y clase a los análisis y campos de disputa. En América Latina, esos mismos años fueron de transformación del escenario político en el ciclo abierto por la Revolución Cubana. Izquierdas de nuevo cuño buscaban radicalizar los procesos de superación de los proyectos nacional desarrollistas que mostraban sus limites y complicidades con el imperialismo. Fue un período marcado por el ascenso de la lucha armada en la mayoría de los países de la región y si bien ya existían militantes y organizaciones feministas, la opresión de las mujeres tendió a ser concebida como una contradicción secundaria, que se resolvería con el triunfo revolucionario, tal como ha

\section{anuario.}


planteado la socióloga chilena, y figura clave del feminismo latinoamericano, Julieta Kirkwood (1986).

Ahora bien, con las diferencias que deben reconocerse entre lo que acontecía en el espacio euro-norteamericano y lo que ocurría en América Latina, consideramos que el feminismo de aquel periodo formó parte de las luchas populares en ascenso, luchas que -sobre todo en el llamado Tercer Mundoserían brutalmente derrotadas. En el caso particular de nuestro continente, fue en el contexto de esas derrotas que se produce una emergencia feminista más nítida que en los años anteriores, ligada a la lucha por los derechos humanos y a las organizaciones populares que hicieron frente a la crisis económica provocada por los ajustes neoliberales de los años ochenta. Años en que se inician los Encuentros Feministas Latinoamericanos y en que circulan un conjunto de materiales de difusión por diversos países del continente. En el centro de las publicaciones feministas se situaban las luchas por la democracia, el vínculo entre autoritarismo y patriarcado, y la defensa de la vida en un doble frente: por una parte, contra el secuestro, la tortura, el asesinato y la desaparición de militantes políticos por parte de las dictaduras; y por otra, en la organización popular para combatir el hambre y la necesidad económica en los años de la crisis provocada por el ajuste estructural.

En la actualidad, el feminismo se levanta en un mundo que no está caracterizado por el avance o las victorias del campo popular sino más bien por la agudización de las consecuencias provocadas por el modelo de acumulación neoliberal que arrasó con las conquistas alcanzadas por la clase trabajadora durante la segunda mitad del siglo XX. Hoy en día, tras cuarenta años de expansión del neoliberalismo a escala global, los efectos sociales, políticos y ecológicos que esta fase de acumulación capitalista ha producido adquieren una magnitud tal que el estado de crisis es insoslayable. Estas condiciones determinan la significación histórica de la emergencia feminista actual. Nancy Fraser (2020) ha elaborado una lectura general de la crisis contemporánea desde una perspectiva feminista que permite apreciar el cuadro en su conjunto y comprender el lugar que ocupa el resurgimiento feminista en este ciclo. En un trabajo de largo aliento, Fraser da cuenta de la configuración de "nuevas geografias de la explotación" producidas por el despliegue neoliberal y su impacto en distintas esferas de la vida social y política. Su análisis sugiere que el movimiento feminista contemporáneo constituye una respuesta a la crisis general del capitalismo, y por tanto, estaría jugando un rol preponderante en la recomposición de fuerzas y actores que podrian desarrollar una política anticapitalista ajustada a las condiciones actuales. 
"Hasta que valga la pena vivir": violencia y reproducción social como claves de la emergencia feminista contemporánea en América Latina

En el contexto latinoamericano, además, la emergencia feminista se produce en un momento político de particular inestabilidad, que oscila entre la crisis del llamado ciclo progresista y el ascenso de gobiernos neoliberales y ultraconservadores; entre estallidos sociales y recrudecimiento de la represión policial y militar; entre el neofascismo y alternativas de democratización empujadas por movimientos y actores sociales heterogéneos. En este escenario convulsionado por tendencias en pugna, el movimiento feminista ha tenido un rol gravitante en la articulación de luchas y en la activación de formas de contestación que, por la masividad que han alcanzado, muestran una enorme capacidad de convocar a la sociedad a la intervención en el espacio público, capacidad que actores tradicionales de la política han perdido ostensiblemente en las últimas décadas.

E1 movimiento feminista latinoamericano, partiendo de la lucha contra la violencia hacia las mujeres en su expresión más brutal como el femicidio, ha activado un conjunto de otras demandas que atraviesan distintas esferas de la vida social, como el trabajo, el medioambiente, la educación y las finanzas, en las que la violencia contra las mujeres se reproduce y las desigualdades de género se ahondan. De este modo, enhebrando la espesa trama de violencias estructurales y desplegando formas de intervención política en distintos niveles, el movimiento feminista en América Latina se ha convertido en una actoría fundamental de las luchas del presente.

\section{El nudo de la violencia como clave de articulación y reivindicación histórica}

Retomando la historia de los feminismos latinoamericanos, la década de los ochenta fue fundamental para la articulación feminista a nivel local. Uno de los puntos que releva Catalina Trebisacce (2020) es la significante violencia que emerge en los discursos de las organizaciones sociales de la época, cuestión que se visibiliza en los Encuentros Feministas Latinoamericanos y del Caribe, desde su primera instancia en Bogotá en 1981. En éste, se articulan algunas demandas y fechas claves para el movimiento latinoamericano, enfatizando el carácter internacional del 8 de marzo en tanto hito de lucha feminista, como también la instauración del 25 de noviembre como fecha que buscaba visibilizar la denuncia contra la violencia hacia las mujeres, al conmemorar el asesinato de las hermanas Mirabal en República Dominica bajo la dictadura de Rafael Leónidas Trujillo. Patsili Toledo recuerda que si bien desde finales de los ochenta ha existido un contexto de fragmentación feminista, la violencia contra las mujeres ha logrado constituirse "en un rasgo común a la experiencia de

\section{anuario.}


todas las mujeres, en todo el mundo, aunque pueda tener muy diversas manifestaciones e intensidades en cada realidad específica" (2018, p. 122), adquiriendo así una característica universal y transversal.

Este eje -el de la violencia-, constituye un aspecto clave de las reivindicaciones feministas contemporáneas, cuyo origen puede fecharse en las organizaciones feministas de los ochenta. El contexto específico de varios países del cono sur durante la década vinculado a un pasado autoritario reciente, o directamente bajo una dictadura militar, posibilitó una reflexión y organización feminista que particularmente problematizó aspectos como la violencia política, de género y la cuestión de la democracia (Vargas, 2008). En este sentido, un primer elemento característico del feminismo latinoamericano radica en la relevancia del trabajo activista feminista en organizaciones no gubernamentales, donde aspectos como la denuncia sobre situaciones de violencia que afectaban a mujeres fueron gravitantes para el desarrollo de una problematización politica desde este aspecto, como también de temáticas vinculadas a la educación, salud sexual y reproductiva y derechos humanos. El contexto latinoamericano favoreció una comprensión de la violencia contra las mujeres en un amplio margen, ya sea cometida por particulares como por agentes estatales. En ambos casos, la cuestión de la violencia contra las mujeres fue considerada como un asunto relativo a los derechos humanos (Toledo, 2018, p. 35).

Julieta Kirkwood, la relevante socióloga y feminista chilena, es clave para comprender la densidad y espesor analítico que adquiere el feminismo en este contexto, considerando la comprensión política de la violencia y su vinculación con el problema autoritario. Específicamente, para Kirkwood, la realidad dictatorial bajo la cual se batía el país significó -paradójicamente- un incentivo para la articulación feminista, toda vez que el feminismo se constituyó como una respuesta radical frente a la dictadura, reclamando el retorno democrático (Kirkwood, 1986). Conocida es la frase acuñada por Kirkwood -"Democracia en el país y en la casa"- slogan representativo del movimiento feminista latinoamericano (Álvarez, 1997; Vargas, 2008), cuyo mensaje resumía la dicotomía entre el espacio público y privado, enfatizando que las prácticas autoritarias, violencias y formas de dominación basadas en el género se desarrollaban en el seno del hogar. Para las feministas de los ochenta, este aspecto fue sustantivo en tanto que posiciona las demandas feministas en vinculación y tensión con una realidad política específica, utilizando la clave de la violencia como posibilidad de unión y vinculación entre agrupaciones, colectivos y centros de pensamiento relativos a las temáticas de las mujeres. 
"Hasta que valga la pena vivir": violencia y reproducción social como claves de la emergencia feminista contemporánea en América Latina

A la vez, el trabajo y producción teórica sobre el tema estuvo estrechamente vinculado con el activismo feminista en la materia. En este contexto, América Latina presenta una particularidad en relación al tratamiento de la temática de violencia contra las mujeres, siendo un rasgo característico la preeminencia del trabajo de organizaciones feministas, activistas y ONG's, quienes posicionaron políticamente la temática, antes que el interés profesional o académico sobre la materia (Hiner, 2019; Toledo, 2018; Trebiscacce, 2020). En los noventa, el alto nivel de coordinación regional del movimiento feminista visibilizado en la realización periódica de los EFLAC, "permitió levantar el fenómeno de los femicidios/feminicidios como uno de los ejes de la acción y denuncia feminista a nivel continental desde fines de la década de 1990" (Toledo, 2018, p. 41). La violencia contra las mujeres funcionó, así como uno de los temas de las agendas feministas que genera mayor adhesión dentro del movimiento, así como de unidad en las demandas y reivindicaciones (Toledo, 2018; Vargas, 2008).

En este sentido, no es de extrañar la vigencia que adquiere la temática en la actualidad. Antes bien, es preciso enfatizar que la condición estructural de la violencia contra las mujeres y cuerpos feminizados ha sido posicionada por el movimiento feminista durante los últimos años, ampliando el marco desde donde se comprendia el término. En un texto reciente, Sandra Palestro y Soledad Rojas de la Red Chilena contra la Violencia hacia las Mujeres, realizan un recuento de las formas de la violencia contra las mujeres y los mecanismos de respuesta desde la experiencia chilena reciente. En él apuntan que es una violencia específica, situada culturalmente y que responde al sentido común, la cual estaría en sintonía con "un patrón cultural androcéntrico y de dominación masculina, presente estructuralmente en la organización del Estado, la sociedad y el mercado, transmitido por todas las instancias de socialización" (2018, p. 327), transversalidad que es posible advertir identificando patrones comunes que permiten establecer conexiones entre sus distintas manifestaciones. Esta perspectiva es crítica respecto de la centralidad y mirada unifocal con que se ha abordado el problema de la violencia contra las mujeres y cuerpos feminizados desde las políticas estatales de los noventa, señalando las falencias del abordaje institucional en tanto la comprende de forma parcial y acotada, centrada en los asuntos de familia o de pareja. Para Palestro y Rojas, esta entrada y circunscripción al problema de la violencia -de carácter asistencialista y fragmentado- posibilita una dependencia y revictimización, "ocultando su carácter político en tanto asunto medular de la discriminación y opresión de las mujeres" (2018, p. 327-328). 
Comprender la centralidad que adquiere la violencia en las formas de discriminación y opresión hacia las mujeres en la actualidad ha sido fundamental para la articulación de movimientos y acciones feministas, dando cuenta del constante y creciente cuestionamiento frente a la violencia en tanto estructura y mecanismo de dominación patriarcal. Este aspecto ha sido clave para situar el problema de la violencia contra las mujeres y cuerpos feminizados desde una perspectiva múltiple e integral. Así, es posible señalar que las situaciones de violencia contra las mujeres que han sido posicionadas públicamente por las organizaciones feministas, constituyen aspectos significativos tanto para la visibilización del problema -y con ello su reconocimiento más explícito- como para fomentar la proliferación de organizaciones y colectivos que se dedican al tema. Un importante ejemplo de lo anterior lo constituyen las movilizaciones feministas estudiantiles que, a lo menos en el caso chileno, visibilizaron situaciones de acoso sexual en el espacio universitario. La movilización del año 2018 se caracterizó por la toma efectiva de establecimientos de educación superior, levantando distintas demandas que iban desde la incorporación de protocolos y medidas para afrontar las situaciones de violencia contra las mujeres y cuerpos feminizados, como también el posicionamiento de aspectos vinculados a la educación no sexista o educación feminista (Follegati, 2018; Zerán, 2018).

La visibilización de las situaciones de violencia en el campo educativo no tardaron en traspasar dicho escenario, señalando una problematización general sobre la condición de las mujeres y cuerpos feminizados cuestión que se graficó en un histórico y multitudinario 8 de marzo del 2019, convocando hasta ese momento la manifestación más masiva desde el retorno a la democracia en Chile. A la vez, muchas de las consignas y problemáticas traspasan fronteras, estableciendo formas similares de manifestación universitaria frente a situaciones de acoso sexual y abuso en ciudades como Bogotá o Ciudad de México (Mingo, 2020; Forero, 2019). En este sentido, es posible señalar que tanto el activismo feminista reciente, como también la proliferación de coordinadoras, organizaciones y colectivos feministas constituyen aspectos significativos que han posibilitado la problematización y posicionamiento de la demanda contra la violencia. Así, al igual que en los ochenta, la clave organizativa constituye un aliciente fundamental para la articulación masiva del feminismo, como también el reconocimiento y legitimación de las demandas. Desde este punto de vista, plantear la violencia contra las mujeres y cuerpos feminizados en clave de derechos ha propiciado una vinculación entre organizaciones feministas de larga data con las nuevas generaciones. Sin embargo, un elemento contemporáneo que puede constituir una particularidad, 
"Hasta que valga la pena vivir": violencia y reproducción social como claves de la emergencia feminista contemporánea en América Latina

radica en el reconocimiento de la violencia dentro un abanico de situaciones que contribuyen a la precariedad y vulneración de derechos, incorporando otras problemáticas al entramado feminista.

\section{La violencia como clave de inteligibilidad}

Al son de "Un violador en tu camino", el colectivo chileno Las Tesis dio a conocer una de las propuestas teóricas de Rita Segato, en relación a las formas que adquiria la violencia de contra las mujeres y cuerpos feminizados. La apuesta del colectivo combinó movimiento, letra y ritmo cuyo contenido buscaba denunciar la vinculación estructural entre violencia y patriarcado. La transversalidad de la performance cruzó fronteras no sólo latinoamericanas, sino que rodeó el globo replicándose en diversas latitudes (desde Lima a Estambul) traduciéndose en distintos idiomas, pero siempre bajo la premisa de que todas quienes participaban de la intervención comprendian el contenido y motivación de la crítica sin importar el dónde, cómo, ni cuándo. Esa transversalidad es uno de los rasgos que podrian caracterizar al movimiento feminista contemporáneo, uno donde la masividad y espontaneidad se articulan como respuesta frente a situaciones de violencia, propiciando una verdadera marea morada que replica un ¡Basta Ya! (Follegati, 2020). Para Verónica Gago, la performance de Las Tesis señala un internacionalismo que funciona a través de la conexión de luchas heterogéneas que articulan un diagnóstico y confrontación colectiva, que "sale de la superficie digital para hacerse cuerpo común en una multiplicidad de espacios y hacerlo como voz colectiva. Ya no es solo texto que escribimos entre todas, sino performatividad compartida que rompe con la sumisión de género, con toda victimización, y se hace presencia tanto como mensaje" (Gago, 2020, p. 16).

Ahora bien, como es sabido, las situaciones de violencia contra las mujeres y cuerpos feminizados han existido históricamente en tanto constituyen una manifestación específica del patriarcado, por lo tanto cabe preguntarse por qué en la actualidad su denuncia concita tal masividad e internacionalismo. Un primer aspecto radica en el contexto e historia latinoamericana, el cual, como hemos visto, posee particularidades específicas que ayudan a la problematización de la violencia contra las mujeres y cuerpos feminizados desde una perspectiva singular, donde se articula la historia colonial del continente, la producción teórica feminista y la problematización de la violencia como un asunto de derechos humanos. 
$\overline{\text { De igual manera, un aspecto que nos interesa resaltar es que el }}$ carácter transfronterizo de la violencia contra las mujeres expresado en la cobertura global de la performance de Las Tesis, así como la consigna "Ni una menos", apelan a una configuración social de la violencia que escapa a situaciones particulares o específicas, manifestándose estructuralmente. El especial eco de la lucha contra los feminicidios en Argentina, Chile o México, apela también a la cuestión latinoamericana para el tratamiento del tema, donde se combinan otros factores que vinculan de forma explícita la violencia colonialista con la patriarcal y la económica de carácter neoliberal. Esta triangulación configuraría un aspecto relevante para la problematización de la violencia contra las mujeres y cuerpos feminizados, como también la activación del movimiento feminista. Para Segato, la violencia contra las mujeres ha tenido una significación sustantiva en la articulación de formas de dominación sobre el cuerpo femenino, dando cuenta de 'marcas de cohesión' o vinculación de un grupo o comunidad a través de la violencia tanto fisica como psicológica, simbólica o institucional. Inscrita en los sistemas jurídicos actuales, la relación entre moralidad y sujeción de la mujer se vincula en la propia construcción legal. Para la antropóloga, la violencia se encausa en una estructura elemental que entra en tensión en la forma irreductible entre el sistema de estatus y de contrato, aspectos correlativos y coetáneos que se combinan desde la prehistoria de la humanidad (Segato, 2003, p. 144). En este escenario, el cuerpo de las mujeres en América Latina se convirtió en un territorio de conquista y disputa: "muestran las violaciones que acompañan la ocupación de territorio en las guerras premodernas y también las modernas, es parte indisociable de una noción ancestral de territorio, que vuelve, una y otra vez, a infiltrarse intrusivamente en el texto y en la práctica de la ley" (Segato, 2003, p. 143).

Esta forma situada de comprender el problema nos enfrenta a la particularidad de producción y reproducción de mecanismos de subyugación y violencia, componiendo un sistema articulado que funciona a través del ejercicio, ocupación y violencia hacia el cuerpo de las mujeres y cuerpos feminizados. Mandato, dirá la antropóloga, que subsiste gracias a la intención moralizadora de reducir y aprisionar a las mujeres bajo todos los mecanismos posibles y existentes. Así, se engarzan violencias sexuales, psicológicas y físicas, como también, el mantenimiento de una estructura y orden capitalista que produce problemas sociales como la actual feminización de la pobreza (Segato, 2003, p. 145). Estos aspectos - por lejos de estar segmentados- nos desafian a una comprensión que releva las 'marcas' diferenciadoras que, tanto el sistema de estatus como de contrato, utilizan para la producción de cuerpos racialiazados, étnicos, feminizados, o precarios. Señalar desde el feminismo una visión 
"Hasta que valga la pena vivir": violencia y reproducción social como claves de la emergencia feminista contemporánea en América Latina

unívoca o unicausal sobre las formas de producción y reproducción de la violencia estructural, sería contraproducente con una visión que comprende a los cuerpos como espacios y campos de disputa política. Sujetos subordinados, racializados e inmigrantes vivencian de forma más explícita las injusticias del sistema económico, jurídico y soberano, y es en ellos donde persisten los efectos de la colonialidad del poder, y se significa con más fuerza la infiltración de la violencia en el contrato y la ley.

El movimiento y la teoría feminista han relevado cómo los crimenes de odio gestionan un ensañamiento frente a cuerpos que representan categorias sociales plausibles de apropiación y de demostración de poder a través de su dominio y posibilidad de muerte (Segato, 2003, p. 253). América Latina, no es sino un territorio donde lo abyecto es la forma común, a través de cuerpos que propician un panorama de rostros desventajados en el devenir neoliberal. Cuerpos en disputa que encarnan precariedad y vulneración. Desde una clave latinoamericana, el énfasis feminista ha estado en comprender cómo dicha violencia estructural se articula con otras contradicciones e intersecciones, en la particularidad que adquiere la violencia en los territorios atravesados por el narcotráfico (Valencia, 2010), por el racismo contra las afrodescendientes o la pobreza y exclusión que atraviesan los pueblos indígenas. Ejemplo de lo anterior fue el asesinato de Marielle Franco en Río de Janeiro el 2018, y el de Berta Cáceres el 2016, en Honduras. Las elaboraciones feministas contemporáneas han logrado discernir continuidades y especificidades históricas del momento neoliberal, propiciando la activación del movimiento a través de la denuncia de tales situaciones. En condiciones como las presentes, una reflexión sobre la violencia y el femicidio debe ser capaz de unir todos los nudos de la trama. Elaboraciones como las de Rita Segato $(2003,2013)$ han abierto un camino en esa dirección al complejizar y situar la reflexión sobre la violencia contra las mujeres y cuerpos feminizados en un contexto acrecentado por las crisis del neoliberalismo. Estos aspectos profundizan las posibilidades de que el movimiento feminista adquiera una profundidad y masividad mayor que en otras latitudes, aspecto que se reforzará por el cuestionamiento a situaciones cotidianas o normalizadas que en la actualidad son comprendidas como parte de la violencia estructural cimentada por el patriarcado. En este sentido, un segundo factor que es relevante de analizar radica en el problema de la reproducción social desde la perspectiva de la violencia contra las mujeres. 


\section{Violencia estructural y crisis de la reproducción social}

Un aspecto sustantivo que ha producido la actual marea feminista ha sido la comprensión en clave de violencia -y por lo tanto una sostenida crítica- de una serie de situaciones profundamente arraigadas en las prácticas culturales y estructuras sociales, ensanchando los marcos de interpretación de las situaciones de violencia en general y ampliando el parámetro y sentido común sobre el tema. El movimiento feminista latinoamericano ha sido fundamental en este proceso, produciendo y entregando herramientas conceptuales para la reinterpretación de la violencia, que constantemente ha enmarcado nuestra lucha en una disputa en clave de derechos (Daich \& Tarduci, 2018). Comprender la violencia contra las mujeres y cuerpos feminizados desde un punto de vista estructural nos desafia a observar el problema desde espacios diferenciados e interconectados de reproducción (laboral, territorial, sexual, salud, ambiental, medios de comunicación, etc.) en los que se producen violencias específicas y al mismo tiempo derivadas de las estructuras socioeconómicas que imperan en las sociedades neoliberales. Este énfasis establece la necesidad de vinculación, articulación y especificidad sin dislocar el foco de atención al analizar la violencia contra las mujeres en cada aspecto de la vida en el que es posible percibir una condición particular de injusticia en cuanto a sus derechos. De este modo, el feminismo permite comprender la condición de injusticia, vulnerabilidad y precarización que viven miles de mujeres en su cotidianidad en cuestiones como el acceso a la salud, la seguridad social o en la doble o triple jornada laboral, a través del desarrollo de la reflexión en torno a las violencias estructurales.

Esta lectura está en la base de las más importantes movilizaciones feministas de estos años. El paro o huelga feminista del 8 de marzo, que se ha levantado como forma de articulación regional, responde así a una acción y lenguaje político que, como señala Verónica Gago, busca encarar la ofensiva femicida desde una multiplicidad de factores. En esta óptica, se reconoce el femicidio no sólo desde un punto de vista doméstico o individualizante, sino, sobre todo, como un extremo en que "se traman y expresan nuevas formas de explotación laboral, violencias económicas, violencias estatales y violencias políticas" (Gago, Gutiérrez, Drapper et.al., 2018, p. 10). En este mismo sentido, en palabras de la mexicana Raquel Gutiérrez, la ola femicida desatada contra las mujeres en México y América Latina "la impugnamos y comprendemos simultáneamente como uno de los vértices de la figura triangular de la expropiación-explotacióndominación contemporánea que se impone a través de la violencia generalizada y la guerra irregular contemporánea" (Gutiérrez en Gago, Gutiérrez, Drapper et.al., 2018, p. 43). 
"Hasta que valga la pena vivir": violencia y reproducción social como claves de la emergencia feminista contemporánea en América Latina

$\overline{\text { En consideración a estos elementos, es plausible sostener que en la actualidad }}$ la cuestión de la violencia contra las mujeres y cuerpos feminizados no sólo recoge un aspecto vinculado a la defensa de la vida, y con ello una continuidad con las luchas sobre DDHH durante los períodos autoritarios, sino que también incorpora el problema de la economización de la reproducción social como clave de dominación y articulación de las formas de violencia contra las mujeres. Este aspecto parece sustantivo para visualizar cómo los distintos escenarios locales y contemporáneos han visto nacer movimientos, organizaciones y colectivos visibilizando la violencia como clave de inteligibilidad de las injusticias tanto simbólicas como económicas y politicas. El rechazo transversal frente a estas situaciones, junto con la consecuente organización y masividad de las manifestaciones, darán cuenta de un aspecto sustantivo para comprender los feminismos actuales, a saber, aquel que entiende y transforma las situaciones de violencia contra las mujeres como un reclamo politico, propiciando procesos de politización a través de su denuncia. Quizás, al igual que en los ochenta, la unidad que concita la denuncia contra la violencia hacia las mujeres como un asunto de derechos humanos configura también una potencia sustantiva del movimiento feminista, esta vez, vehiculizando el descontento en una pluralidad de espacios representados por el carácter transversal de la violencia estructural. Al mismo tiempo, esta ampliación de lo que se entiende por violencia ha conducido necesariamente a una problematización general de las condiciones de existencia en esta fase de desarrollo neoliberal, marcada por la precarización creciente de las condiciones de vida de la clase trabajadora global con las especificidades que la explotación adquiere para las mujeres, cuerpos feminizados y racializados. El feminismo, en este sentido, opera extendiendo los marcos de comprensión a partir de los cuales se interpreta la realidad social que somete a su escrutinio, a su diagnóstico y a su proyecto transformador abarcando cada vez más dimensiones (Gago, 2020; Gago \& Cavallero: 2019).

\section{Del trabajo doméstico al problema general de la reproducción social}

El giro hacia un concepto estructural de violencia y hacia el problema de la reproducción social se produce como respuesta a condiciones concretas del presente. Por una parte, al hecho insoslayable de encontrarnos en medio de una crisis de reproducción social y cuidados que alcanza escala planetaria, y, por otra, a la también creciente proliferación de conflictos sociales asociados a las luchas en la esfera de la reproducción que están en el centro de la conflictividad social en este ciclo, y que en un gran porcentaje están lideradas por mujeres y activistas feministas.

Ahora bien, más allá del protagonismo de estas temáticas en las elaboraciones feministas contemporáneas, es preciso recordar que han tenido un recorrido

\section{anuario.}


largo en la historia del movimiento. La llamada de atención acerca del lugar estratégico del trabajo reproductivo en el sostenimiento de la sociedad capitalista, así como la crítica a la distinción entre producción y reproducción y a las consecuencias políticas de desconocer la condición de trabajadoras a las mujeres dedicadas a las labores domésticas, fueron aportes de enorme trascendencia que realizaron las feministas de los años setenta. Autoras como Isabel Larguía (1972), Margaret Benston (1969), Peggy Morton (1971), Mariarosa Dalla Costa y Selma James (1975) y Silvia Federici (2013 [1975]), desplegaron una contundente elaboración en torno al trabajo doméstico, presentándolo como una pieza clave en la reproducción del capital, en tanto trabajo no pagado y no reconocido como tal. En nuestro continente, la intelectual argentina radicada en Cuba, Isabel Larguía, acuñó el concepto de "trabajo invisible" (1972) y problematizó acerca de la persistencia de la división sexual de trabajo en la sociedad cubana en pleno proceso de transformación revolucionaria, junto John Dumoulin (Larguía \& Dumoulin, 1976). La crítica de esta generación de feministas se dirigía explícitamente a las izquierdas, a partidos, sindicatos y a la teoría marxista en general que había obliterado completamente esta cuestión. Era una crítica teórica, en tanto develaba un vacío de elaboración y una incorrecta comprensión de un mecanismo básico de la sociedad capitalista y era también una crítica política a la jerarquización interna de la clase trabajadora entre quienes reciben un salario por su trabajo y quienes lo realizan de manera gratuita, Federici conceptualizó esta idea como "patriarcado del salario" (Federici, 2018). El aporte hecho en estos años por estas intelectuales y activistas marcó el derrotero de buena parte del pensamiento feminista posterior, que asumió la tarea de profundizar el esclarecimiento de la trabazón existente entre patriarcado y capitalismo y de desarmar la división equívoca entre producción y reproducción.

En la actualidad, a estas formulaciones ya clásicas, se han ido añadiendo un conjunto de elaboraciones en torno a todos aquellos ámbitos de la vida social que forman parte del engranaje de sistemas necesarios para la reproducción de las personas y comunidades (salud, educación, vivienda, pensiones) y que se han convertido en fuentes de extracción de valor, ya sea por la mercantilización de los servicios sociales básicos, la desposesión de territorios para faenas extractivas o el endeudamiento de los hogares ante la insuficiencia de salarios. Desde una perspectiva feminista y marxista, Nancy Fraser (2020) sostiene que en esta fase del capitalismo se produce una combinación de factores que provocan una aguda crisis de reproducción social: el sensible retroceso en las conquistas alcanzadas por el movimiento obrero en el siglo XX, un debilitamiento sostenido de la democracia y la capacidad de orientar la acción política hacia los intereses de las mayorías, así como una privatización y mercantilización de la reproducción social y los cuidados. 
"Hasta que valga la pena vivir": violencia y reproducción social como claves de la emergencia feminista contemporánea en América Latina

Las consecuencias de este avance neoliberal en términos de crisis de reproducción social han vuelto a poner esta cuestión en el centro de las reflexiones de la teoría crítica y del feminismo en particular. Se puede apreciar que muchas autoras, siguiendo distintos caminos, concluyen cuestiones similares en relación al carácter de la crisis, al entramado entre capitalismo, extractivismo, colonialismo y patriarcado, y a las potencialidades politicas que contienen las luchas en la esfera de la reproducción en esta etapa histórica. Silvia Federici (2010) y Wendy Brown (2016), enfatizan en cómo el cuerpo de las mujeres se dispuso en tanto mecanismo de aseguramiento y disciplina para solventar el trabajo productivo, bajo la posibilidad misma de reproducción del modelo económico a partir de las tareas de reproducción y cuidado. David Harvey (2004), retomando el análisis marxiano de la acumulación originaria, instaló la lectura de la acumulación por desposesión como una clave para comprender los mecanismos de la fase neoliberal del capitalismo. En una línea similar, Federici (1990) y su colectivo Midnight Notes (1990) han hablado de los "nuevos cercamientos" para referirse a las asonadas del capital contra los bienes comunes. En América Latina, dado el impacto de las politicas extractivistas en el continente y la cantidad de conflictos socioambientales provocados por la acción de grandes corporaciones privadas y estatales en territorios que son defendidos por sus comunidades, la clave de lectura de la acumulación por desposesión ha sido particularmente productiva (Galafassi, 2012). Paralelamente, las elaboraciones agrupadas bajo el rótulo de "Teoría de la Reproducción Social" están poniendo el foco en cuestiones como la educación, la salud pública, los sistemas de pensiones y la seguridad social en general (Batthacharya, 2017; Ferguson, 2020; Ferguson, \& McNally, 2013; Arruza, Bhattacharya \& Fraser, 2019). En un sentido similar, la economía feminista ha contribuido a instalar una lectura feminista y anticapitalista de una serie de problemas que actualmente están en el centro de las discusiones acerca de cómo enfrentar la crisis en una dirección que permita orientar la organización social al sostenimiento de la vida (Perez Orozco, 2014; Carrasco, 2017). Asimismo, los feminismos indigenas y comunitarios (Gargallo, 2015; Espinosa, Gómez, Ochoa, 2014), los ecofeminismos (Mies \& Shiva, 2016) y el pensamiento post extractivista (Svampa y Viale, 2014; Gudynas, 2019), realizan diagnósticos y propuestas para superar este modelo de desarrollo que juzgan incompatible con el mantenimiento de la vida humana y la naturaleza. Todas estas elaboraciones, que en la actualidad están en pleno florecimiento, apuntan desde miradas diversas a un mismo problema: la crisis general producida por el neoliberalismo y la necesidad de reorientar la organización social de forma tal que la reproducción de la vida esté en el centro y no se subordine a la ley del valor.

\section{Las luchas de la vida contra el capital en clave feminista}

\section{anuario.}


Además de este conjunto de elaboraciones, la emergencia de un abanico de luchas sociales ancladas en la esfera reproductiva, convierte a esta dimensión de la vida social en un elemento ineludible en la reflexión teóricopolítica. Imprimiendo una novedad respecto a las grandes luchas que protagonizaron el siglo XX, en este ciclo, a las tradicionales peleas en el campo del trabajo, se han sumado los conflictos sociales vinculados a la defensa de las condiciones de vida: salud, pensiones, educación, bienes naturales comunes, etc. No es casual que en América Latina exista un elevado número de asesinatos a dirigentes sociales vinculados a luchas contra el extractivismo o que estallidos sociales como el que sacudió a Chile, país laboratorio de las políticas neoliberales desde fines de los años setenta, hayan tenido como causa profunda el malestar social acumulado ante la mercantilización extrema de la vida y las desigualdades cada vez más acentuadas (Ferretti \& Dragnic, 2020; Ruiz, 2020). La conflictividad social hoy gira, en gran parte, en torno a la recuperación de la soberanía personal y colectiva sobre las condiciones de reproducción social. Las movilizaciones más importantes y masivas a nivel continental se han producido en este plano, por lo que la reproducción adquiere, en los hechos, un rol estratégico en las luchas contra el capital.

Al interior de estas luchas, el feminismo constituye actualmente la fuerza con mayor poder articulador y con mayor capacidad de elaborar una lectura global de la imbricación entre patriarcado, capitalismo, violencia estructural y crisis de la reproducción social. Al mismo tiempo, se aprecia cómo las luchas concretas libradas en diversos territorios y frentes, están lideradas principalmente por mujeres y activistas feministas. Incluso en los sectores más masculinizados de las organizaciones sociales, como el sindical, la proporción de mujeres y de feministas en dirigencias y bases ha ido creciendo. En Chile y Argentina, los dos países latinoamericanos que han tenido las movilizaciones feministas más masivas de este ciclo, se observa con claridad cómo el movimiento feminista está cruzando la mayoría de las luchas sociales del presente y, además, está elaborando las lecturas más capaces de articular una perspectiva global para entender la vinculación de todas las violencias. Los documentos desarrollados en el seno del feminismo son contundentes en su diagnóstico sobre la relación entre patriarcado y capitalismo. El colectivo Ni Una Menos en Argentina, por ejemplo, desde el primer llamado a Huelga Internacional Feminista en 2017, expuso una serie de elementos que dan cuenta de las dimensiones estructurales de las violencias contra las mujeres y cuerpos feminizados. En el manifiesto que convoca a la movilización podemos leer: "Paramos para denunciar: Que el capital explota nuestras economías informales, precarias e intermitentes. Que los Estados nacionales y el mercado nos explotan cuando nos endeudan. Que los Estados criminalizan nuestros movimientos migratorios. Que cobramos menos que los varones y que la brecha 
"Hasta que valga la pena vivir": violencia y reproducción social como claves de la emergencia feminista contemporánea en América Latina

salarial llega, en promedio, al 27\%. Que no se reconoce que las tareas domésticas y de cuidado son trabajo que no se remunera y suma, al menos, tres horas más a nuestras jornadas laborales. Que estas violencias económicas aumentan nuestra vulnerabilidad frente a la violencia machista, cuyo extremo más aberrante son los femicidios" (NUM, 2017a). En Argentina, asimismo, ha sido insistente la crítica feminista a la financiarización de las economías domésticas y a los ajustes o recortes de la inversión pública en derechos sociales. "Las finanzas -sostiene el mismo Colectivo-, a través de las deudas, constituyen una forma de explotación directa de la fuerza de trabajo, de la potencia vital y de la capacidad de organización de las mujeres en las casas, en los barrios, en los territorios. La violencia machista se hace aún más fuerte con la feminización de la pobreza y la falta de autonomía económica que implica el endeudamiento. El movimiento de mujeres se consolidó como un actor social dinámico y transversal capaz de poner en escena las diversas formas de la explotación económica. Dejamos de ser meramente víctimas justamente porque podemos hacer comprensibles las formas en que nos explotan y accionar colectivamente contra los múltiples despojos" (NUM, 2017b).

En Chile, las elaboraciones feministas avanzan en la misma dirección. Por ejemplo, la Coordinadora Feminista 8M, una de las más importantes a nivel nacional, ha levantado la consigna "Mujeres trabajadoras a la calle contra la precarización de la vida" como el lema principal de las convocatorias a las huelgas feministas los últimos 8 de marzo. En un documento de 2018, redactado pocos días después de la movilización, leemos: "nos convocamos de forma articulada desde distintos espacios sociales de resistencia y lucha clasista, antirracista y feminista para manifestarnos en una jornada de protesta en contra de la precarización de la vida situándonos desde el mundo del trabajo. Donde los bajos sueldos, la subcontratación, y la carencia de derechos sociales, sexuales y reproductivos aumenta el agobio de las mujeres, pues somos las que debemos asumir, por regla general, el trabajo doméstico y los cuidados en las familias, sin reconocimiento, de manera gratuita y cargando con el costo creciente de las vidas entregadas en todas sus esferas al mercado" (CF8M, 2018). Los ejemplos podrían multiplicarse. En distintos territorios del continente encontramos elaboraciones que apuntan a elementos similares, así como un sinnúmero de luchas sociales en las que activistas feministas se hallan desplegadas.

Se puede afirmar, entonces, que la esfera de la reproducción social se ha convertido el escenario principal de las luchas de la vida contra el capital (Ferretti \& Dragnic, 2020) y que el feminismo es la fuerza articuladora más transversal en este conjunto de luchas populares. La reflexión sobre la violencia estructural y el problema de la reproducción social en clave feminista adquieren 
por ello importancia estratégica en la tarea de articular en la sociedad polos antineoliberales que den proyección a estos conflictos en un contexto en que las estructuras tradicionales de representación de las clases populares, como partidos de izquierda y sindicatos, se encuentran sensiblemente debilitadas (Fraser, 2020). Se aprecia así, cómo el feminismo, en tanto teoría y práctica política, adquiere un estatuto privilegiado en las condiciones actuales debido a su capacidad de ofrecer una lectura global de la crisis actual y, al mismo tiempo, debido a su enorme capacidad de movilización de masas.

\section{Conclusiones}

La trayectoria larga del movimiento feminista en la región, a partir de los años setenta, establece un importante antecedente para el movimiento actual, al articular una plataforma reivindicativa que se fue desplegando durante las siguientes décadas. Durante los noventa y 2000, las constantes campañas para la denuncia sobre los femicidios cobraron una importante relevancia al visibilizar en el espacio público la radicalidad y transversalidad de éstos en tanto crimenes de odio, uniendo luchas, consignas y manifestaciones desde Santiago de Chile a Ciudad de México. Asumir el carácter transversal y global de la violencia contra las mujeres y cuerpos feminizados ha constituido históricamente un factor sustantivo para la articulación del movimiento feminista, particularmente en un escenario local donde las violaciones a los derechos humanos y las vulneraciones producto de las inestabilidades políticas, narcotráfico o pobreza, conjugan aspectos que acrecientan aún más las situaciones de violencia. Desde nuestra perspectiva, la particularidad del momento actual radica en que la denuncia frente a la violencia física y simbólica por razón de género levantada por el movimiento feminista desde los años setenta y de los ochenta con mayor fuerza y articulación en el continente, apela a una comprensión cada vez mayor de los marcos con que se analiza y comprende la violencia, enfatizando en el carácter estructural y transversal de ésta.

A la vez, el movimiento contemporáneo revisita problemas y demandas históricas del movimiento de los ochenta, actualizando las consignas y reivindicaciones que han sido levantadas por las feministas de la región, demandas que operan como una transversalidad transfronteriza que funciona como una denuncia, y a la vez, politiza a sectores de mujeres cada vez más amplios y transversales de la sociedad. Las problemáticas sobre violencia contra las mujeres y reproducción social, fueron contenidos cuya profundidad y elaboración posee un momento anterior, anclados en la historicidad del movimiento. Esta característica señala que si bien presenciamos una 
"Hasta que valga la pena vivir": violencia y reproducción social como claves de la emergencia feminista contemporánea en América Latina

visibilización de las demandas, muchas de ellas corresponden a una reflexión que venía desarrollándose pero que entró en un cierto suspenso. En este recorrido, el movimiento feminista latinoamericano ha logrado trastocar los contornos de los proyectos políticos nacionales, siendo hoy en día una posibilidad para comprender cómo desde el movimiento -de carácter internacionalista- se cuestionan las formas tradicionales y las claves locales con que se configura el cuadro político democrático a espaldas de las mujeres. E1 feminismo, en tanto teoría y acción, vendría a copar el sentido político debilitado de clase dirigente, resignificando problemáticas históricas pero conjugándolas en una clave de derechos humanos, sociales y politicos. Movimiento que construye un escenario donde las demandas de las mujeres y cuerpos feminizados ocupan la calle y la movilización masiva como mecanismo de presión, señalando una actoría colectiva que no duda en cuestionar -una y otra vez- el hastío frente a un orden político y económico que reinventa constantemente las recetas de la exclusión y explotación de las mujeres, reedificando las claves de la subordinación patriarcal.

A la vez, el movimiento feminista contemporáneo es una respuesta masiva a los problemas más urgentes que aquejan al mundo de hoy, problemas que se comprenden desde la violencia estructural en tanto prisma que permite comprender el ensañamiento transversal de la violencia de los cuerpos de las mujeres y cuerpos feminizados, articulando la precarización propia del neoliberalismo con la cotidianeidad impuesta por el patriarcado. El carácter masivo y convocante que tiene el movimiento responde así a las condiciones sociales sentidas como intolerables por las mujeres y por franjas importantes de la población, definiendo y señalando como abuso situaciones y prácticas que no tenían una visibilización desde ese registro, más bien, tendían a ocultarse como prácticas culturalmente construidas. En este sentido, el feminismo en tanto teoría, reflexión y movimiento, ha logrado calar en el sentido común al ofrecer diagnósticos críticos sobre las actuales condiciones de vida, nombrando situaciones y experiencias que no habian sido identificadas como vulneraciones, despojos o violencias. Por esta razón las actuales convocatorias latinoamericanas a la conmemoración del 8 de marzo han posicionado manifestaciones llamando a la Huelga feminista o Paro, visibilizando el trabajo doméstico no remunerado, como también aspectos que traspasan el ámbito de género pasando a concebirlas en una dimensión estructural.

Dentro de esta lectura estructural, revisitar la articulación entre capitalismo y patriarcado ha sido uno de los aspectos característicos de los nuevos espacios feministas. Como hemos señalado, este impetu ha levantado antiguas consignas, pero complementándolas con prácticas políticas y repertorios de acción que permiten poner nuevos problemas en el espacio público y sacudir, 
con enormes convocatorias, los escenarios políticos. El problema de la reproducción social se vislumbra como una clave sustantiva para la proyección del movimiento feminista en los años venideros, como también una real alternativa para la coordinación regional en clave antineoliberal. Desde ahí, el movimiento feminista puede apostar a una transformación sustantiva de las estructuras sociales, siendo claro el carácter radical y categórico de la necesidad de transformación. La relevancia teórica adquirida por la problemática de la reproducción social, pero sobre todo el carácter gravitante que han tenido "las luchas por la vida", es decir, por derechos sociales, por el medioambiente, por el acceso al agua, por la recuperación territorial, adquieren importancia estratégica para proyectar una política anticapitalista. De todas esas luchas el movimiento de mujeres forma parte, y el feminismo, en tanto conjunto de elaboraciones teórico políticas, ha desarrollado una capacidad de lectura y sistematización que ha contribuido al fortalecimiento y a la auto comprensión de esas mismas luchas.

Por todo lo anteriormente expuesto, se puede sostener que la emergencia feminista contemporánea constituye uno de los procesos políticos más relevantes y desafiantes del presente ciclo histórico en América Latina. Presenciamos un período con una importante capacidad de convocatoria y movilización, pero también uno donde los espacios de articulación regional parecen ser fluctuantes o esporádicos. Las movilizaciones feministas responden más a hitos específicos, estallidos, donde, a partir de situaciones concretas, se exige justicia en casos de violencia contra las mujeres, o bien de "legalidad" refiriéndonos a las demandas por el aborto libre, seguro y gratuito. Sin embargo, se aprecia una inclinación a la denuncia que no siempre es acompañada de una voluntad de intervenir de manera activa en la formulación de propuestas en el campo de la política pública y las leyes. El feminismo tiene una enorme capacidad de movilización, y sus convocatorias masivas impactan en el escenario social y político, pero hasta el momento, no ha existido una capacidad instalada para participar en la elaboración de propuestas políticas en el espacio institucional. Esto se debería a varias razones. Por una parte, la elevada proporción de mujeres que acuden a las manifestaciones no lo hace de forma organizada, sino más bien como individualidades, lo que implica una dificultad en el ejercicio de representación o 'traducción' de las demandas feministas en el plano institucional. Otra, es la reticencia de los sectores más radicales o autónomos del feminismo organizado a hacerse parte de instancias que impliquen diálogos con instituciones gubernamentales. Predomina en estos espacios una comprensión de la acción politica feminista como denuncia, agitación, protesta y trabajo a nivel local, excluyendo el trato con la institucionalidad politica, al que se apela más bien para impugnarlo. Elaborar esta dimensión es un desafio para el movimiento feminista contemporáneo, 
"Hasta que valga la pena vivir": violencia y reproducción social como claves de la emergencia feminista contemporánea en América Latina

tanto a nivel académico como activista, cuestión que hace fundamental la necesidad de indagar en los caminos del feminismo, enfatizando en las preguntas y propuestas estratégicas que avizoran las diferentes corrientes y agrupaciones.

Finalmente, un elemento crucial en este punto es que en estas batallas se han sentido convocados amplios sectores sociales: no sólo mujeres y no sólo feministas. En el estallido social chileno, por ejemplo, fue un pueblo heterogéneo que sin dudas representaba a la mayoría de la población, el que irrumpió en las calles (Grau, Follegati \& Aguilera, 2020; Follegati, 2020). Esta esfera de luchas por recuperar soberanía colectiva sobre las condiciones de existencia, es un elemento fundamental en la recomposición de un sujeto popular que, conservando su heterogeneidad irreductible, pueda ir desarrollando formas organizativas para hacer avanzar sus intereses y sus propias propuestas acerca de cómo debe organizarse la sociedad, distribuir la riqueza y el poder político, y todas aquellas cuestiones que afectan la vida colectiva. A fin de cuentas, la consigna "Hasta que valga la pena vivir" apela a la lucha por recuperar soberanía sobre la vida, lo que implica también replantearse el problema de la democracia y cómo las mayorias sociales se han ido expresando en revueltas espontáneas y en manifestaciones multitudinarias como las que el movimiento feminista ha logrado convocar. Revueltas que pueden dar paso a la construcción del poder suficiente para superar el neoliberalismo y orientar la vida al bienestar social y no a la valorización del valor. En definitiva, el movimiento de mujeres y el feminismo tienen, y pueden tener todavía más, un rol gravitante en la recomposición de un campo popular capaz de hacer prevalecer la vida frente a la devastación a la que nos conduce el proyecto neoliberal, extractivista y patriarcal. Un movimiento feminista que articule la pluralidad de luchas latinoamericanas, reestableciendo su radicalidad expresiva y potencia emancipadora.

\section{Bibliografia}

Almeida, P. (2020). Movimientos sociales: la estructura de la acción colectiva. Buenos Aires: Clacso.

Álvarez, S. (1997). Articulación y transnacionalización de los feminismos latinoamericanos. Debate Feminista. (15)

Arruza, C.; Bhattacharya, T.; Fraser, N. (2019). Feminismo para el 99\%. Un manifiesto. Martínez: Rara Avis.

\section{anuario.}


Benston, M. (1969). The Political Economy of Women's Liberation. Monthly Review (21)

Bhattacharya, T. (2017). Social Reproduction Theory. Remapping Class, Recentering Oppression. Londres: Pluto Press.

Brown, W. (2016). Pueblo sin atributos. La secreta revolución del neoliberalismo. Barcelona: Malpaso.

Carrasco, B. (2017). La economía feminista. Un recorrido a través del concepto de reproducción. Ekonomiaz (91).

CF8M [Coordinadora Feminista 8M] (2018). "Hacia una agenda común de movilización contra la precarización de la vida". En https:/ / www.facebook.com/notes/coordinaci\%C3\%B3n-8-de-marzo/haciauna-agenda-com\%C3\%Ban-de-movilizaci\%C3\%B3n-contra-laprecarizaci\%C3\%B3n-de-la-vida/2293614130865358/. Visitado el 10 de septiembre de 2020.

Daich, D. \& Tarducci, M. (2018). De feminismos y violencias. Recuperar la historicidad de las luchas para enfrentar nuevos desafios. En Mujeres y feminismos en movimiento. Buenos Aires: EFFL, Editorial de la Facultad de Filosofia y Letras.

Dalla Costa, M., James, S. (1975). El poder de las mujeres y la subversión de la comunidad, México: Siglo XXI.

Espinosa Miñoso, Y; Gómez Correal, D; Ochoa Muñoz, K (eds.) (2014). Tejiendo de otro modo: Feminismo, epistemología y apuestas descoloniales en Abya Yala. Popayán: Universidad del Cauca.

Federici, S. (1990). The debt crisis, Africa and the New Enclosures. Midnight Notes (10).

Federici, S. (2010). Calibán y la bruja. Madrid: Traficantes de Sueños.

Federici, S. (2013). "Salarios contra el trabajo doméstico". En Federici, S. La revolución en punto cero. Madrid: Traficantes de sueños.

Federici, S. (2018) El patriarcado del salario. Críticas feministas al marxismo. Buenos Aires: Tinta Limón.

Femenías, M. L. et. al. (2015). Violencias cruzadas. Miradas y perspectivas. Rosario: Protohistoria Ediciones. 
"Hasta que valga la pena vivir": violencia y reproducción social como claves de la emergencia feminista contemporánea en América Latina

Ferguson, S. (2020). Women and work. Feminism, Labour, and social reproduction. Londres: Pluto Press.

Ferguson, S. \& McNally, D. (2013). "Capital, fuerza de trabajo y relaciones de género". Presentación a la edición de Historical Materialism de Lise Vogel, Marxism and the opression of women. Toward a Unitary Theory, Brill: LeidenBoston. $\quad$ pp. xvii-xl. Recuperado: https:/ / marxismocritico.com/2017/01/16/capital-fuerza-de-trabajo-yrelaciones-de-genero/

Ferretti, P; Dragnic, M. (2020). Revolt in Chile. Life against capital. Viewponit Magazine. Recuperado de https:/ /www.viewpointmag.com/2020/02/13/revolt-in-chile-life-againstcapital/

Follegati Montenegro, L. (2020) "Nos quitaron hasta el miedo": Los feminismos en la revuelta social chilena. En LASA FORUM, Fall 2020. Vol. 51: 4. Pp. 4-10

Follegati Montenegro, L. (2018). El feminismo se ha vuelto una necesidad: movimiento estudiantil y organización feminista (2000-2017). Anales de la Universidad de Chile (14)

Follegati Montenegro, L. (2019). "Violencia estructural, precarización y feminismo: apuntes para una discusión”. En Violencia Estructural y feminismo: apuntes para una discusión. Santiago de Chile: Ed. Red Chilena contra la Violencia hacia las mujeres. Pp. 17-28.

Forero, S. (2019). Ante las violencias contra universitarias: acción colectiva, estudiantil y feminista. Nómadas (51).

Fraser, N. (2020). Los talleres ocultos del capital. Un mapa para la izquierda. Madrid: Traficantes de Sueños.

Gago, V. (2014). La razón neoliberal. Economias barrocas y pragmática popular. Buenos Aires: Tinta Limón.

Gago, V. (2019). La potencia feminista o el deseo de cambiarlo todo. Buenos Aires: Tinta Limón.

Gago, V; Malo, M; Cavallero, L. (2020). La Internacional Feminista: luchas en los territorios y contra el neoliberalismo. Buenos Aires: Tinta Limón. 
Gago, V., Cavallero, L. (2019) Una lectura feminista de la deuda. "Vivas, libres y desendeudadas nos queremos". Buenos Aires: Fundación Rosa Luxemburgo.

Gago, V.; Gutiérrez Aguilar, R; Draper, S; Menéndez, M; Montanelli, M; Bardet, M; Rolnik, S. (2018). 8M Constelación feminista. Buenos Aires: Tinta Limón.

Galafassi, G. (2012). Entre viejos y nuevos cercamientos La acumulación originaria y las politicas de extracción de recursos y ocupación del territorio. Theomai (26). Recuperado de http://www.revistatheomai.unq.edu.ar/NUMERO\%2026/Galafassi\%20-\%20Entre\%20viejos\%20y $\% 20$ nuevos\%20cercamientos.pdf

Gargallo, F. (2015). Feminismos desde Abya Yala. Ideas y proposiciones de las mujeres de 607 pueblos en Nuestra América. México, D. F.: UAM.

Grau, O., Follegati, L. \& Aguilera, S. (2020). Escritos Feministas en tiempos de revuelta. Santiago de Chile: Lom.

Gudynas, E. (2019). Extractivismos y corrupción. Anatomía de una intima relación. Quito: Abya Yala.

Harvey, D. (2004). El nuevo imperialismo. Madrid: Akal.

Hiner, H. (2019). Violencia de género, Pobladoras y feminismo popular. Santiago de Chile: Tiempo Robado.

Kirkwood, J. (1986). Ser politica en Chile. Las feministas y los partidos. Santiago de Chile: Flacso.

Larguía, I. (1972). Contra el trabajo invisible. En La liberación de la mujer. Año cero, Buenos Aires: Granica.

Larguía, I. \& Dumoulin, J. (1976). Hacia una ciencia de la liberación de la mujer, Barcelona: Anagrama.

Melucci, A. (1996). Challenging Codes. Collective Action in the Information Age. Cambridge: Cambridge University Press.

Midnight Notes Collective. (1990). The New Enclosures. Boston: Midnight Notes. Mies, M; Shiva, V. (2016). Ecofeminismo. Madrid: Traficantes de Sueños.

Mingo, A. (2020). El tránsito de estudiantes universitarias hacia el feminismo. Perfiles Educativos (167). 
"Hasta que valga la pena vivir": violencia y reproducción social como claves de la emergencia feminista contemporánea en América Latina

Morton, P. (1971). A Woman's Work is Never Done. En Altbach, E. (dir.) From Feminism to Liberation. Cambridge: Schenkman Publishing.

Ni Una Menos (2017a) Llamamiento al Paro Internacional de Mujeres - 8 de marzo 2017. http://niunamenos.org.ar/manifiestos/llamamiento-al-parointernacional-de-mujeres-8-de-marzo-2017/ Visitado el 10 de septiembre de 2020.

$\mathrm{Ni}$ Una Menos (2017b) \#DesendeudadasNosQueremos http://niunamenos.org.ar/manifiestos/desendeudadasnosqueremos / visitado el 10 de septiembre de 2020.

Osborne, R. (2009) Apuntes sobre violencia de género. Barcelona: Ed. Bellaterra.

Palestro, S. \& Rojas, S. (2018). Violencia hacia las mujeres: un problema estructural. Revista Anales de la Universidad de Chile (4).

Perez Orozco, A. (2014). Subversión feminista de la economía. Aportes para un debate sobre el conflicto capital-vida. Madrid: Traficantes de Sueños.

Pleyers, G. (2018). Movimientos sociales en el siglo XXI: perspectivas y herramientas analíticas. Buenos Aires: Clacso.

Ruiz, C. (2020). Octubre chileno. La irrupción de un nuevo pueblo. Madrid: Taurus.

Segato, R. (2003). Las estructuras elementales de la violencia: contrato y estatus en la etiología de la violencia". En Segato, R. Las Estructuras Elementales de la Violencia. Buenos Aires: Universidad Nacional de Quilmes.

Segato, R. (2013). La escritura en el cuerpo de las mujeres asesinadas en Ciudad Juárez. Buenos Aires: Tinta Limón.

Svampa, M; Viale, E. (2015). Maldesarrollo. La Argentina del extractivismo y del despojo. Buenos Aires: Katz.

Tarrow, S. (1989). Democracy and Disorder: Protest and Politics in Italy, 19651975. Oxford: Oxford University Press

Toledo, P. (2015). Movimiento de mujeres, derechos humanos y tipificación del femicidio/feminicidio en Latinoamérica. En Femenías, M, et. al. Violencias cruzadas. Miradas y perspectivas. Rosario: Protohistoria Ediciones. 
Touraine, A. (1985). An introduction to the study of social movements. Social Research (54).

Trebisacce, C. (2020). Un nacimiento situado para la violencia de género. Indagaciones sobre la militancia feminista porteña de los años 80. Anacronismo e Irrupción (10).

Vargas, V. (2008) Feminismos en América Latina. Su aporte a la política y a la democracia. Colección Transformación Global. Lima: Programa Democracia y Transformación Global, Fondo Editorial Universidad Nacional Mayor de San Marcos, Flora Tristán Ediciones.

Zerán, F. (2018). (Ed.) Mayo Feminista. La Rebelión contra el patriarcado. Santiago, Chile: LOM.

Recibido: 3 de Agosto de 2020

Aceptado: 17 de Septiembre de 2020 Versión Final: 2 de Noviembre de 2020 